International Journal of Modern Physics: Conference Series

(C) World Scientific Publishing Company

\title{
EXTRACTIONS OF POLARIZED AND UNPOLARIZED PARTON DISTRIBUTION FUNCTIONS
}

\author{
P. JIMENEZ-DELGADO \\ Thomas Jefferson National Accelerator Facility \\ 12000 Jefferson Ave., Newport News, VA 23606, USA \\ pedro@jlab.org
}

Received 18 August 2013

\begin{abstract}
An overview of our ongoing extractions of parton distribution functions of the nucleon is given. First JAM results on the determination of spin-dependent parton distribution functions from world data on polarized deep-inelastic scattering are presented first, and followed by a short report on the status of the JR unpolarized parton distributions. Different aspects of PDF analysis are briefly discussed, including effects of the nuclear structure of targets, target-mass corrections and higher twist contributions to the structure functions.

Keywords: QCD; parton distributions; spin.

PACS numbers: 12.38.-t, 12.38.Bx
\end{abstract}

\section{Introduction}

The quantitative description of high-energy collisions involving hadrons in the initial state relies on the fact that in such interactions the hadronic structure, in terms of their constituent quarks and gluons (partons), may be embodied by universal parton distribution functions (PDFs). Clearly, a precise knowledge of these functions is of fundamental intrinsic interest. In addition, their accurate determination is of crucial importance because they are often one of the leading sources of uncertainty in the results. Due to the inherent nonperturbative effects in a QCD bound state like the proton, the PDFs cannot be obtained by perturbative calculations, but are usually extracted from experimental information. An interplay between theoretical and experimental developments is necessary for this, and it is currently limited by the existence and availability of data and of a variety of calculations and tools for parton distribution analysis.

In a first approximation the parton distribution functions of quarks and gluons of given helicity can be taken (at a particular resolution scale) to depend only on the momentum (fraction) of the parton parallel to that of the hadron to which it belongs (parent). Thus additional degrees of freedom, namely the transverse components of the parton momentum as well as spatial dependences, are disregarded (integrated out). This defines the so-called collinear approximation, which has been 
developed over the last few decades by the world community and provides the base for most of the analyses at current accelerators, for example searches for new physics phenomena which are being carried out at the LHC.

Current determinations of (spin-averaged or unpolarized) parton distributions are based to a great degree on deep-inelastic-scattering (DIS) data, from HERA and from fixed-target experiments. Existing data from hadron colliders also contribute important information, because of their complementary kinematics as well as their different dependence on the parton types. In a similar way the spin-dependent or helicity parton distribution functions constitute the basis for the description of experiments in which the spin direction of the initial hadrons is known (polarized). Spin and orbital angular momentum are essential elements of the nucleon structure from which precise information is relatively (as compared to the unpolarized case) scarce at the moment. A wealth of data on the angular momentum of quarks will be available in the near future from the $12 \mathrm{GeV}$ upgrade of Jefferson Lab. Because of the capabilities of CEBAF accelerator at Jefferson Lab in measuring small cross sections at extreme kinematics, its data are particularly useful for studying the large momentum fraction region, and are complementary to measurements at hadron colliders, namely at LHC and RHIC.

A comprehensive review on parton momentum and helicity distributions in the nucleon has recently been given in Ref. 1 . Here we briefly overview our ongoing extractions of polarized $\left(\mathrm{JAM}^{2}\right)$ and unpolarized $\left(\mathrm{JR}^{3}\right)$ parton distribution functions of the nucleon. A description of our general methodology can be found somewhere else ${ }^{22,23,6}$, the emphasis here will be on several corrections and improvements recently included in our analyses, specifically the effects of nuclear corrections and higher-twist contributions to the structure functions.

\section{Polarized PDFs: JAM}

The JAM (Jefferson Lab Angular Momentum) Collaboration is a new enterprise involving theorists and experimentalists from the nuclear and hadron physics community to study the angular-momentum-dependent structure of hadrons broadly understood. In relation with these analyses an interactive database containing the world data on polarized scattering experiments is being developed and is publicly available ${ }^{7}$.

A first major goal is to perform a global next-to-leading order (NLO) analysis of spin dependent DIS structure functions and asymmetries, paying particular attention to the high- $x$ and low- $Q^{2}$ regions. The importance of obtaining reliable parton distribution fits at large $x$ is manyfold. Accurate PDFs at large $x$ are necessary to reduce the uncertainty on QCD backgrounds in searches for new physics in high- $p_{T}$ spectra, or for new heavy particles at the LHC. At the other end of the spectrum, the behavior of quarks at $x \rightarrow 1$ reflects the nonperturbative structure of the nucleon, with PDF ratios such as $d / u, \Delta u / u$ or $\Delta d / d$ sensitive to the interquark dynamics at long distances ${ }^{1,8}$. Finally, the uncertainty in the extraction of 
the gluon helicity PDF at small $x$, which can be obtained from forward particle production in polarized $p p$ collisions, is fundamentally limited by the uncertainty on the large- $x$ PDF of the quark against which the gluon scatters.

Including large- $x$ and low- $Q^{2}$ data in a global fit requires careful consideration of various finite- $Q^{2}$ corrections to perturbative calculations, such as those arising from target mass and higher twist effects, as well as nuclear corrections associated with the use of Deuteron and ${ }^{3} \mathrm{He}$ targets (which are necessary for $u$ and $d$ quark flavor separation). These have been shown in recent unpolarized fits to be important to correctly describe DIS data at low invariant mass $W^{9,10,11,3}$ and are even more important in polarized fits, where low- $W$ DIS data comprise a substantial fraction of the available data.

To study the impact of large- $x$ and low- $Q^{2}$ effects in polarized fits, we begin with a baseline fit which makes use of inclusive DIS data only. The fits presented here include two kinds of $\mathcal{O}\left(1 / Q^{2}\right)$ corrections: target mass corrections (TMCs) and higher twist (HT) contributions. TMCs are treated in the OPE formalism ${ }^{12}$, while higher twist terms are fitted to data. In contrast to other global fits, we include a complete treatment of HT corrections for both the $g_{1}$ and $g_{2}$ structure functions within the same analysis. This has been previously investigated for $g_{1}$ or $g_{2}$ separately ${ }^{13,14,15,16}$, while it is considered here in the context of a simultaneous global PDF fit to both $g_{1}$ and $g_{2}$ data.

To account for the nuclear corrections, previous polarized global analyses have relied exclusively on the effective polarization ansatz, in which the polarized PDF in the nucleus, $\Delta f_{i}^{A}$, is related to the polarized PDFs in the proton and neutron by $\Delta f_{i}^{A} \approx\langle\sigma\rangle^{p} \Delta f_{i}^{p}+\langle\sigma\rangle^{n} \Delta f_{i}^{n}$, with $\langle\sigma\rangle^{p(n)}$ the average polarization of the proton (neutron) in the nucleus. The JAM analysis is the first attempt to systematically incorporate the effects of nuclear binding and Fermi motion in deuterium and ${ }^{3} \mathrm{He}$ 17,18. Corrections due to the struck nucleon being off-shell will be included in subsequent analyses, but are expected to play a less important role than for unpolarized PDFs ${ }^{10}$ because of the generally larger uncertainties on the polarized DIS data at high $x$.

The last important methodological aspect of the JAM fits is the use of the measured parallel and perpendicular $A_{\perp}$ asymmetries, rather than reconstructed structure functions, and the calculation of $F_{2}$ and $F_{L}$ from an unpolarized fit using a common set of theoretical corrections ${ }^{3}$. This allows one to benefit from the largest possible unpolarized data set covering the same kinematics, and avoids attributing to polarized PDFs effects neglected in the unpolarized fits.

The DIS data set considered in the fits presented here include polarized DIS measurements from SLAC, SMC, COMPASS, HERMES and Jefferson Lab on proton, deuterium and ${ }^{3} \mathrm{He}$ targets. Perturbative calculations are performed in Mellin moment space. The leading twist contribution $g_{1}^{(\tau=2)}$ is calculated at NLO, and for $g_{2}^{(\tau=2)}$ we use the Wandzura-Wilczek relation. Higher twist contributions to proton and neutron structure functions are included according to 

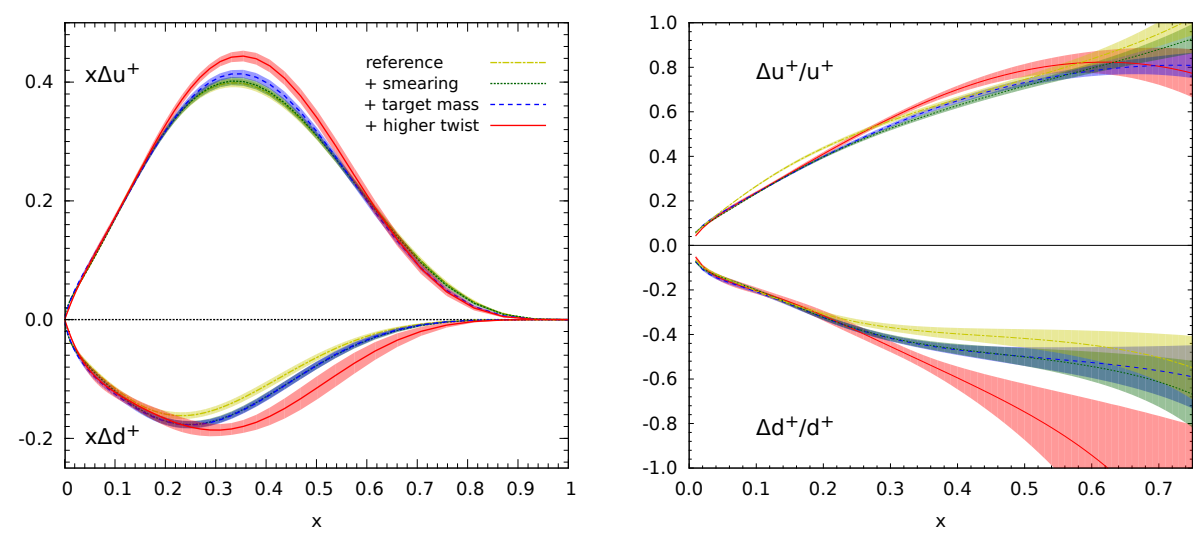

Fig. 1. (Left) Polarized $x \Delta u^{+}$and $x \Delta d^{+}$distributions and their uncertainties for the JAM13 PDF set at $Q^{2}=1 \mathrm{GeV}^{2}$, illustrating the effects of the nuclear smearing (green dotted), target mass (blue dashed) and higher twist (red solid) corrections, relative to the reference fit (yellow dot-dashed). (Right) Corresponding ratios of polarized to unpolarized distributions.

$$
\begin{aligned}
& g_{1}=g_{1}^{(\tau=2)}+g_{1}^{(\tau=3)}+g_{1}^{(\tau=4)} \\
& g_{2}=g_{2}^{(\tau=2)}+g_{2}^{(\tau=3)}
\end{aligned}
$$

where a twist-3 contribution to $g_{1}$ is determined by the twist-3 part of $g_{2}{ }^{12}$,

$$
g_{1}^{(\tau=3)}\left(x, Q^{2}\right)=4 x^{2} \frac{M^{2}}{Q^{2}}\left(g_{2}^{(\tau=3)}\left(x, Q^{2}\right)-2 \int_{x}^{1} \frac{d y}{y} g_{2}^{(\tau=3)}\left(x, Q^{2}\right)\right) .
$$

The parametrization for the function $g_{2}^{(\tau=3)}$ has been inspired by the calculations of Braun et al. ${ }^{19}$, and a spline approximation is used for $g_{1}^{(\tau=4)}\left(x, Q^{2}\right)=h(x) / Q^{2}$. The QCD evolution of $h$ and $g_{2}^{(\tau=3)}$ is for now neglected, which seems reasonable given the precision of the experimental data.

The distributions at the initial scale $\mu_{0}=1 \mathrm{GeV}^{2}$ are parametrized as

$$
x \Delta q^{+}\left(x, \mu_{0}\right) \equiv x(\Delta q+\Delta \bar{q})=N_{q} x^{a_{q}}(1-x)^{b_{q}}\left(1+A_{q} x+B_{q} x\right),
$$

for $q=u, d$. The DIS data are not sensitive to the sea quark distributions $\Delta \bar{u}$ and $\Delta \bar{d}$, and only marginally (due to the different evolution) to $\Delta \bar{s}$, so a fixed shape determined by counting rules at large $x$, and reproducing the behavior of the $\Delta u$ and $\Delta d$ quark PDFs at small $x$, was adopted. The normalizations of the quark distributions were determined from baryon decays. Although $\Delta g$ formally contributes to DIS at second order and through QCD evolution, in practice the data give rather mild constraints. For the results presented here a fixed reasonable shape was used for $\Delta g$ at the input scale.

In Fig. 1, we sequentially study the effect of the various theoretical corrections on the $\Delta u^{+}$and $\Delta d^{+}$distributions starting from a fit with no TMC and HT corrections and using the effective polarization approximation without smearing for nuclear 

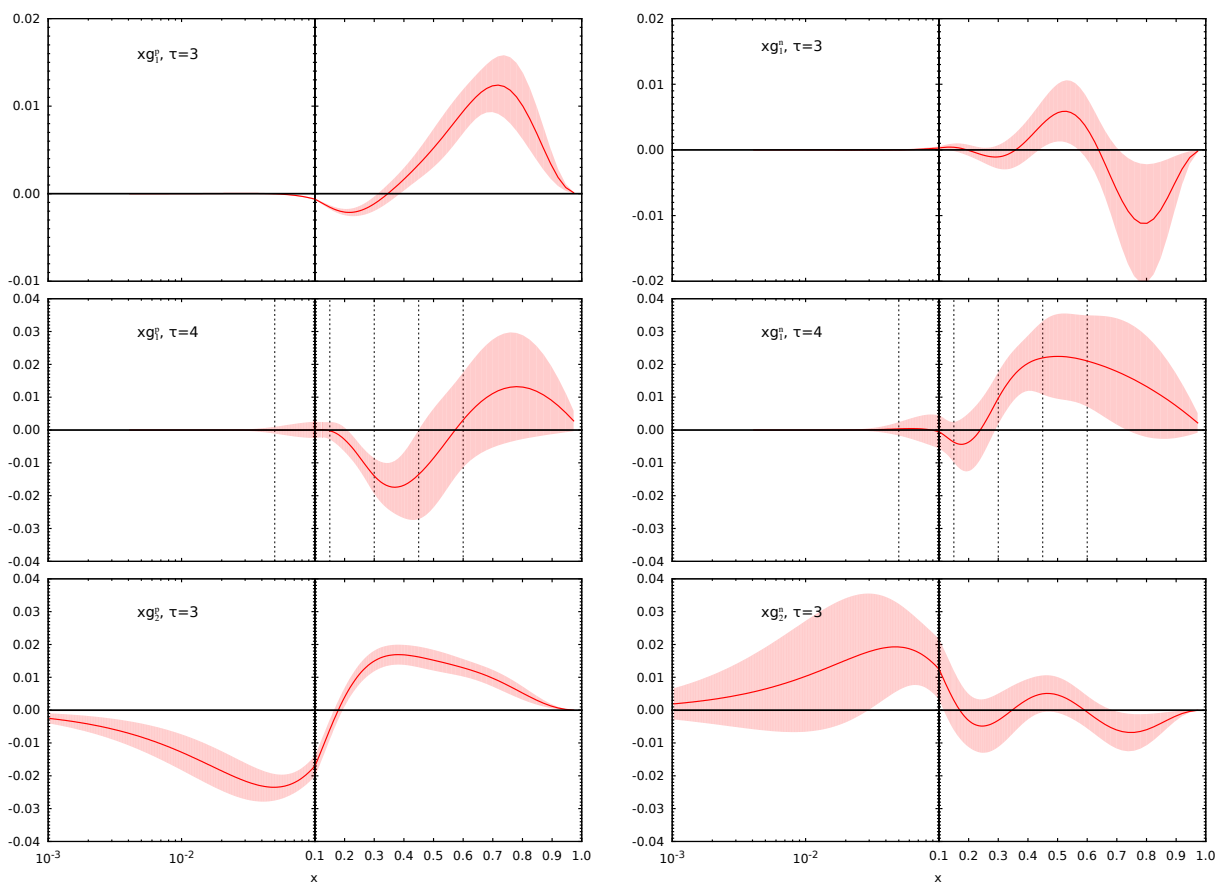

Fig. 2. The proton ((left) and neutron (right) higher twist terms determined in the JAM fit.

targets. At small values of $x$ the effects of the theoretical corrections is negligible; however, at $x \gtrsim 0.3$ they can give up to $20 \%$ corrections for $\Delta u^{+}$and more than $50 \%$ corrections for $\Delta d^{+}$(the relative correction can be even larger at $x \gtrsim 0.8$, although the PDFs are not constrained in this region).

The same effects are more clearly illustrated through the polarization ratios $\Delta u^{+} / u^{+}$and $\Delta d^{+} / d^{+}$, where the unpolarized distributions are fitted within the same theoretical framework as the polarized ${ }^{3}$, thereby eliminating bias arising from assumptions about spin-averaged PDF input. In the intermediate- $x$ region the ratios for both $u$ and $d$ quarks are generally consistent with the symmetric quark model expectations, with the $\Delta u^{+} / u^{+}$increasing towards unity at larger $x$. The $\Delta d^{+} / d^{+}$ratio, on the other hand, remains negative for all $x$ where it is constrained, and shows no indication of the upturn predicted by the helicity conservation models ${ }^{20,21}$. The nuclear and finite- $Q^{2}$ effects can significantly impact the limiting behavior as $x \rightarrow 1$, and clearly additional data are needed to constrain their $x$ dependence at high $x$.

The extracted HT terms are presented in Fig. 2, which clearly demonstrates that it is possible to simultaneously extract the HT contributions to both $g_{1}$ and $g_{2}$ from the present data. The $g_{1}^{(\tau=3)}$ term is clearly non-negligible, and impacts the extraction of the twist- 4 term at $x \gtrsim 0.3$. For the proton, we observe qualitative agreement with $g_{1}^{(\tau=4)}$ extracted by Leader et al. ${ }^{13}$, and with $g_{2}^{(\tau=3)}$ obtained by 
Accardi et al. ${ }^{16}$ and Blümlein and Böttcher ${ }^{15}$. For the neutron the results differ from those of Leader et al. ${ }^{13}$ for $g_{1}^{(\tau=4)}$. This may be due to the nuclear smearing corrections considered in the JAM fits, which substantially modify the extracted $\Delta d^{+}$quark distribution. The neutron contribution to $g_{2}^{(\tau=3)}$ is largely compatible with zero, in contrast to Ref. 16. The Jefferson Lab E99-117 data are found to have a large impact on the neutron's $g_{1}$ fit, and practically determine its twist- 4 contribution.

To summarize, the first results from the JAM Collaboration on polarized PDF fits demonstrate the importance of an accurate treatment of nuclear corrections, i.e., of taking into account the nuclear smearing effects neglected in the more common treatments in terms of effective polarizations. These corrections have the largest impact on the $\Delta d^{+}$distribution. Furthermore, after subtraction of target mass corrections, a simultaneous fit of the higher twist contributions to the $g_{1}$ and $g_{2}$ structure functions is possible and necessary.

Work in progress includes a detailed study of the impact of more recent Jefferson Lab data on the leading twist and higher twist distributions at high $x$. The longer term plans include the analysis of semi-inclusive DIS data and polarized protonproton asymmetries from RHIC.

\section{Unpolarized PDFs: JR}

During the time passed since the last determination of our unpolarized PDFs at $\mathrm{LO}$ and $\mathrm{NLO}^{22}$, and at $\mathrm{NNLO}^{23}$, there have been experimental and theoretical developments which deserve consideration. From the experimental side, besides results coming from LHC which we will not discuss here, new/combined data have been available from HERA and Jefferson Lab. For the older fixed-target data in our analyses we use now directly the measured cross-sections (instead of the extracted structure functions) since potential problems with some of these extractions have been pointed out ${ }^{9}$. A wealth of deuteron data have also been included, for which description we use the nuclear corrections of ${ }^{10}$. Further theoretical improvements include the use of the running mass definition for DIS heavy quark production ${ }^{24}$. Another aspect of our analyses in which we have been working is a more complete treatment of the correlated systematic uncertainties of the data. The least-squares estimator that we use and the careful treatment of multiplicative errors have been given in detail in the appendix Ref. 25.

Another issue raised by the ABM collaboration is the necessity of including higher twist for the description of fixed target data ${ }^{26}$; even if moderate kinematic cuts are used to select the data included in the fits; in particular for SLAC and NMC data. Higher twist (HT) contributions are introduced in the present investigations through the following phenomenological parametrizations:

$$
F_{i}^{\mathrm{HT}, N}\left(x, Q^{2}\right)=F_{i}^{\mathrm{TMC}, N}\left(x, Q^{2}\right)+\frac{h_{i}^{N}(x)}{Q^{2}}
$$

where $i=2, L$ and $N=p, n$. The function $h$ is a cubic spline which interpolates 

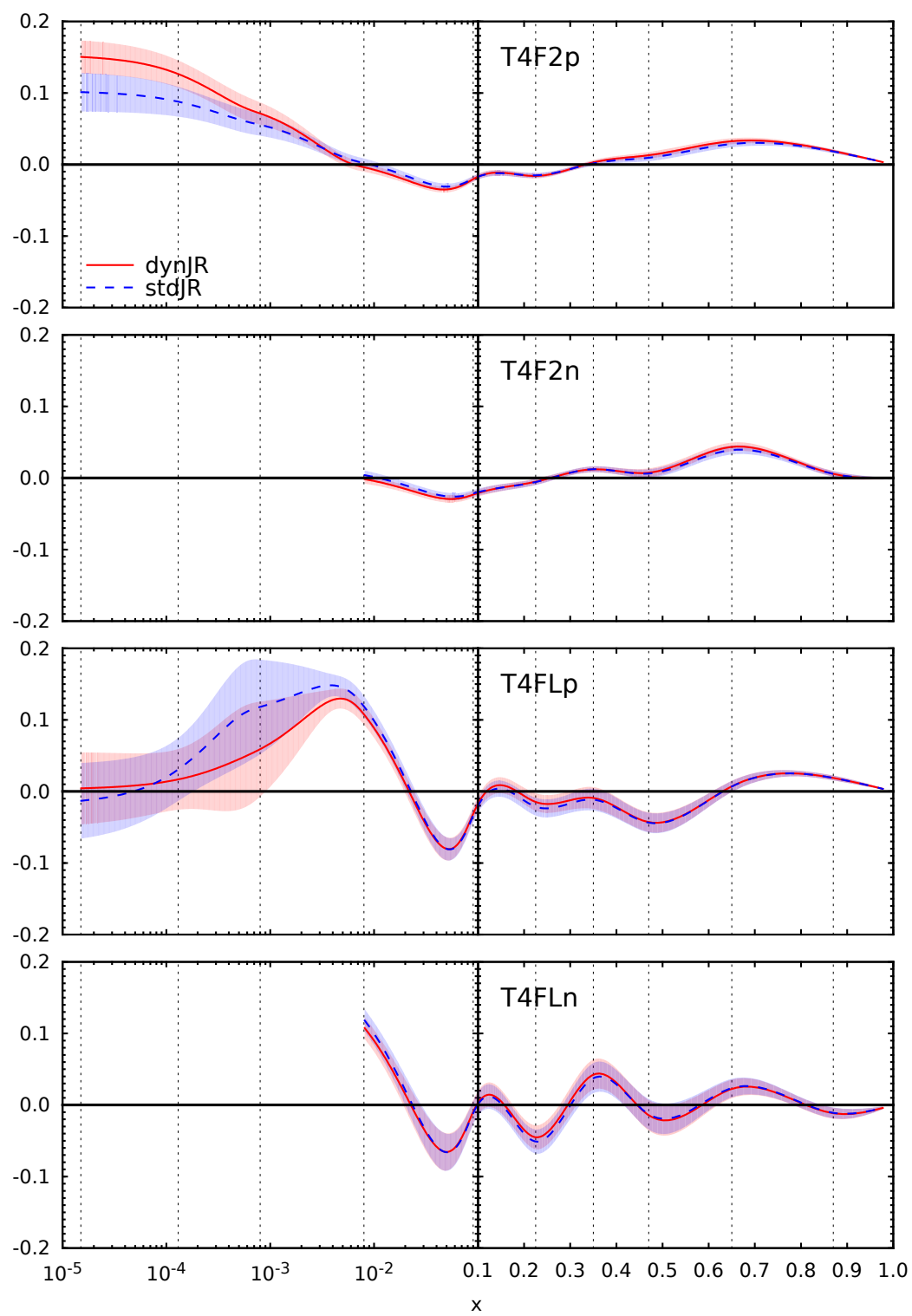

Fig. 3. The proton and neutron higher twist contributions to the structure functions at $Q^{2}=1 \mathrm{GeV}^{2}$ as determined in our current dynamical (dyn) and standard (std) JR analyses, i.e., using $Q_{0}^{2}=0.7 \mathrm{GeV}^{2}$ and $Q_{0}^{2}=1 \mathrm{GeV}^{2}$ respectively.

between a set $\left\{x_{j}, h\left(x_{j}\right)\right\}$ of points, with $x_{j}$ chosen according to the distribution of the data. A possible scale dependence of the $h$ functions has been neglected. The results for these functions are shown in Fig. 3. 
We include the value of the strong coupling constant $\alpha_{s}\left(M_{Z}^{2}\right)$ as a free parameter in our fits. In the current NNLO analyses we find values ranging between about 0.113 for an input scale of $Q_{0}^{2}=0.7 \mathrm{GeV}^{2}$ (dynamical) to about 0.114 for larger input scales (standard). The role of the input scale in parton distribution analyses has been studied in detail in Ref. 25. This agrees rather well with our previous JR09 results ${ }^{23}$. As a matter of fact, dispite the considerable changes in data and in their theoretical treatment, the changes in the parton distributions the we are finding are quite modest.

\section{Conclusions}

We have presented an overview of our ongoing extractions of parton distribution functions of the nucleon. JAM results on the determination of spin-dependent parton distribution functions from world data on polarized deep-inelastic scattering illustrate the importance of an accurate treatment of nuclear corrections, i.e., of taking into account the nuclear smearing effects neglected in the more common treatments in terms of effective polarizations. Moreover, the inclusion of highertwist contributions to the polarized structure functions results in very significant shifts in the parton distributions. In the unpolarize case we have discussed some aspects of the ongoing update of the JR distributions, in particular the inclusion of higher-twist contributions to unpolarized structure functions and our preliminary results for the strong coupling. Despite considerble changes most results are rather consistent with our previous analyses.

\section{Acknowledgments}

I thank W. Melnitchouk, A. Accardi, A. Prokudin, E. Reya, S. Alekhin, J. Blümlein, H. Bötcher for discussions. This work was supported by DOE contract No. DEAC05-06OR23177, under which Jefferson Science Associates, LLC operates Jefferson Lab.

\section{References}

1. P. Jimenez-Delgado, W. Melnitchouk and J. F. Owens, J. Phys. G 40, 093102 (2013).

2. P. Jimenez-Delgado, A. Accardi and W. Melnitchouk, in preparation.

3. P. Jimenez-Delgado and E. Reya, in preparation.

4. M. Glück, P. Jimenez-Delgado and E. Reya, Eur. Phys. J. C 53 355, (2008).

5. P. Jimenez-Delgado and E. Reya, Phys. Rev. D 79, 074023 (2009).

6. B. Lampe and E. Reya, Phys. Rept. 332, 1 (2000).

7. JAM Collab. (P. Jimenez-Delgado et al.), http://www.jlab.org/jam

8. W. Melnitchouk and A. W. Thomas, Phys. Lett. B 377, 11 (1996).

9. S. Alekhin, J. Blümlein and S. Moch, Eur. Phys. J. C 71 (2011) 1723.

10. J. F. Owens, A. Accardi and W. Melnitchouk, Phys. Rev. D 87, 094012 (2013).

11. A. D. Martin et al., Eur. Phys. J. C 73, 2318 (2013).

12. J. Blümlein and A. Tkabladze, Nucl. Phys. B 553, 427 (1999).

13. E. Leader, A. V. Sidorov and D. B. Stamenov, Phys. Rev. D 82, 114018 (2010). 
14. J. Blümlein and H. Böttcher, Nucl. Phys. B 841, 205 (2010).

15. J. Blümlein and H. Böttcher, arXiv:1207.3170 [hep-ph].

16. A. Accardi et al., JHEP 0911 (2009) 093.

17. S. A. Kulagin and W. Melnitchouk, Phys. Rev. C 77, 015210 (2008).

18. S. A. Kulagin and W. Melnitchouk, Phys. Rev. C 78, 065203 (2008).

19. V. M. Braun et al., Phys. Rev. D 83, 094023 (2011).

20. R. Blankenbecler and S. J. Brodsky, Phys. Rev. D 10, 2973 (1974).

21. G. R. Farrar and D. R. Jackson, Phys. Rev. Lett. 35, 1416 (1975).

22. M. Glück, P. Jimenez-Delgado and E. Reya, Eur. Phys. J. C 53, 355 (2008).

23. P. Jimenez-Delgado and E. Reya, Phys. Rev. D 79, 074023 (2009).

24. S. Alekhin and S. Moch, Phys. Lett. B 699, 345 (2011).

25. P. Jimenez-Delgado, Phys. Lett. B 714301 (2012).

26. S. Alekhin, J. Blümlein and S. Moch, Phys. Rev. D 86, 054009 (2012). 